\title{
ANÁLISIS
}

\section{INFONOMISTA: UNA DISCUSIÓN ACTUALIZADA}

\section{Concept of infonomist: an up to date discussion}

\author{
Josep Cobarsí-Morales, Agustí Canals y Eva Ortoll
}

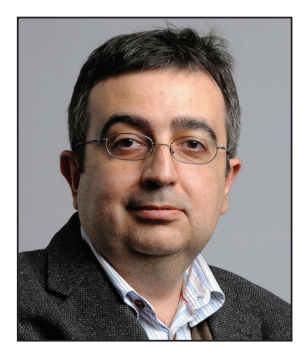

Josep Cobarsí-Morales es ingeniero de telecomunicación por la Universitat Politècnica de Catalunya y doctor en organización de empresas por la Universitat de Girona. Profesor de los Estudis de Ciències de la Informació i de la Comunicació de la Universitat Oberta de Catalunya (UOC) desde 2000, fue director del grado de información y documentación (2007-2015). Investigador del grupo Knowledge and Information Management in Organisations (KIMO) de la UOC (grupo consolidado reconocido por la Generalitat de Catalunya), sus temas de investigación se centran en los sistemas de información, redes sociales e inteligencia competitiva.

http://orcid.org/0000-0002-4382-1058

jcobarsi@uoc.edu

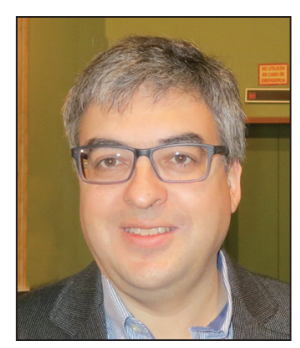

Agustí Canals es profesor de los Estudis de Ciències de la Informació i de la Comunicació de la Universitat Oberta de Catalunya (UOC). Licenciado y master en física por la Universitat Autònoma de Barcelona (UAB) y doctor en administración y dirección de empresas (Management sciences) por Esade (Univ. Ramon Llull, URL). Dirige el grupo de investigación Knowledge and Information Management in Organisations (KIMO) sobre gestión de la información y el conocimiento en las organizaciones. Sus líneas de investigación giran en torno a la gestión estratégica del conocimiento, gestión de información, los procesos de creación de conocimiento científico e innovación, las redes sociales y los sistemas complejos.

http://orcid.org/0000-0002-1700-3983

acanalsp@uoc.edu

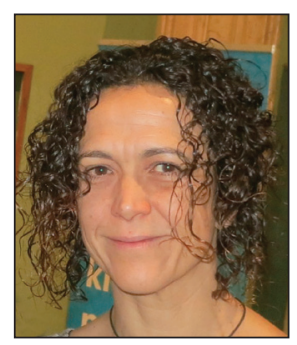

Eva Ortoll es profesora agregada en los Estudis de Ciències de la Informació i de la Comunicació de la Universitat Oberta de Catalunya (UOC), e investigadora del grupo KIMO (Knowledge and Information Management in Organisations) de la misma universidad. Doctora en sistemas de información y documentación por la Universidad de Zaragoza, licenciada en documentación por la Universidad Carlos III de Madrid y diplomada en biblioteconomía por la Universitat de Barcelona. Sus temas de investigación se centran en la inteligencia competitiva, redes de colaboración e intercambio de información, comportamiento de usuarios y gestión del conocimiento. Participa regularmente en proyectos de investigación competitivos.

http://orcid.org/0000-0002-1698-1347

eortoll@uoc.edu

Universitat Oberta de Catalunya, Estudios de Ciencias de la Información y la Comunicación Rambla de Poblenou, 156. 08018 Barcelona, España http://estudios.uoc.edu/es/grados/informacion-documentacion/presentacion http://kimo.uoc.edu

\section{Resumen}

Se plantea una discusión actualizada del concepto de infonomista. Para ello, en primer lugar se recupera la propuesta original de infonomista (e infonomía), esbozada por Cornella en 1998 y se apuntan sus implicaciones. En segundo lugar, se recopilan y comentan definiciones del término publicadas posteriormente. Finalmente, se discuten los siguientes aspectos: la combinación de conocimiento científico de la información actualmente disperso en varias disciplinas, la evolución del significado de información en el discurso científico y en el lenguaje coloquial, y el posicionamiento de diversos actores y colectivos. Todo ello conlleva establecer una selección de potencialidades y retos para el futuro desarrollo y valorización del concepto de infonomista, y su proyección académica y profesional. 


\title{
Palabras clave
}

Gestión de la información; Información y documentación; Infonomía; Disciplinas científicas; Sectores profesionales; Profesionales de la información; Infonomistas; Terminología.

\begin{abstract}
An updated description of infonomist is presented. To do this, we first recover the original proposal of infonomist (and infonomy), sketched by Cornella in 1998. Second, we collect and discuss definitions of the term published subsequently. Finally, we present a description of an infonomist, taking into account the current context: the combination of scientific knowledge on information currently dispersed in various disciplines, the evolution of the meaning of information in scientific discourse and in colloquial speech, and the positioning of various actors and groups. This discussion establishes a selection of potential challenges for future development and strengthening of the concept of infonomist in both academic and professional environments.
\end{abstract}

\section{Keywords}

Information management; Information science; Infonomics; Scientific fields; Profesional sectors; Information professionals; Infonomists; Terminology.

Cobarsí-Morales, Josep; Canals, Agustí; Ortoll, Eva (2016). "Infonomista: una discusión actualizada”. El profesional de la información, v. 25, n. 2, pp. 255-261.

http://dx.doi.org/10.3145/epi.2016.mar.12

\section{Introducción}

El concepto "infonomista" y, relacionado con este, "infonomía", fueron propuestos por primera vez en español por Alfons Cornella (1998). Entonces y ahora el dinamismo, las perspectivas y los problemas, y una considerable confusión, eran características acusadas de las disciplinas y profesiones relacionadas con la información, tanto en nuestro país como en el contexto internacional (Bates, 2015; MoreiroGonzález, 2014; Moreiro-González, 2015; Moreiro-González et al., 2008; Anglada, 2014; Ortiz-Repiso; Calzada-Prado; Aportela-Rodríguez, 2013; Marchionini; Moran, 2012; Lawson; Kroll; Kowatch, 2010). Por ello vale la pena a día de hoy recuperar y revisar los conceptos de infonomista e infonomía, y discutirlos a la luz de las tendencias actuales. En primer lugar se revisa el origen de estos conceptos, y en segundo lugar se da cuenta de su potencial proyección. Ello da paso a una discusión actualizada, y finalmente se presenta una tabla a modo de resumen.

\section{Origen del concepto}

En una nota en la revista El profesional de la información, Cornella (1998) aboga por la conveniencia de "plantear un término más sucinto que describa lo que hace un gestor de información" para que se aplique al conjunto de estos profesionales. Y apunta la solución a partir de la formulación del término "infonomía", entendida como "gestión de la información" o "gestión del bien información", es decir "administración recta y prudente de la información en una organización". Y en consecuencia propone la palabra "infonomista" para designar a quien es capaz de ejercer como gestor de información. Todo ello como analogía respecto al concepto de economía, entendida esta como "administración recta y prudente de los bienes de la casa", según definición del Diccionario de la Real Academia Española de la Lengua citada por el mismo autor.

Al esbozar los conceptos de infonomista e infonomía en esa nota, es importante señalar las implicaciones que el propio Cornella apunta:
En primer lugar se pretende designar un conjunto de competencias, un ámbito de conocimiento, susceptible de proyectarse profesionalmente en diferentes puestos de trabajo concretos, bajo el hilo conductor de la pericia requerida en ellos para su desempeño respectivamente sobre una o diversas facetas de la gestión de información en un determinado contexto organizativo. Es decir, un infonomista así conceptualizado idealmente puede ejercer de archivero, bibliotecario, community manager, content curator, documentalista de empresa, etc. Podría ejercer esos puestos de forma similar a un físico o un economista, quienes pueden proyectar su competencia en el ejercicio de puestos de trabajo muy diversos.

\section{El concepto original de infonomista pro- pone definir un conjunto de competen- cias sobre gestión de información}

En segundo lugar se entiende que para ello debería existir, en el caso de la infonomía, un corpus de conocimiento científico disciplinar establecido y reconocible, como de hecho existe en los casos de la física y de la economía. Y que esta nueva disciplina científica integraría una combinación de conocimientos dispersos hoy en varias disciplinas, las cuales han ido apareciendo históricamente y tratando la información como objeto de estudio desde diversos puntos de vista. Esta disciplina integrada no existe en la actualidad. Más bien, como apunta Bates (2015), hay un conglomerado de disciplinas surgidas de la formalización científica y académica de diferentes puestos de trabajo que han ido apareciendo, en un primer momento vinculados a instituciones (bibliotecas, archivos y museos), y más adelante a partir del ejercicio de ciertos roles en un contexto de empresa u organización genérica (documentalista, content curator, community manager, gestor del conocimiento, etc.). A ese abanico de disciplinas podemos añadir la creciente importancia del concepto de información en ciencias como la física, la biología o la economía, que han ido desarrollando en paralelo 
este concepto atendiendo a sus propias necesidades de conocimiento científico (Ma, 2013; Boisot; Canals, 2004). Acaso el futuro podría deparar una integración de todas esas disciplinas en la sugerida infonomía, pero la realidad actual del conocimiento científico dista de llegar a ese estadio.

En este sentido cabe señalar que, por una parte, la idea originalmente propuesta por Cornella es potencialmente más inclusiva, más abstracta y más científica que la mera formalización de la práctica profesional en un determinado contexto. No obstante, el propio desarroIlo que plantea posteriormente este autor (2000), orientado a la práctica en un contexto empresarial, puede prestarse a equívocos en cuanto a la idea original propuesta.

Hasta aquí hemos expuesto los conceptos de infonomista e infonomía y las ideas fuerza asociadas, partiendo de la propuesta original esbozada por el autor. Veamos ahora qué recorrido ha tenido el término hasta hoy y los significados que se le atribuyen.

\section{Proyección actual}

El término infonomía aparece recogido en lengua catalana por el centro de terminología Termcat, que propone una definición bastante exhaustiva y detallada de infonomía entendida como gestión de información en una organización:

“Infonomía: Técnica que se basa en la selección, la interpretación, el análisis y la distribución de la información en una organización, y en el diseño y el desarrollo de sistemas de información en el ámbito de las nuevas tecnologías".

El propio Termcat apunta como equivalentes los siguientes términos:

-en catalán: gestió de la informació;

-en español: infonomía;

-en francés: gestion de l'information;

-en inglés: information management.

No obstante, infonomía no aparece en español en el Diccionario de la Real Academia, ni tampoco consta en la versión española de Wikipedia. En diccionarios generales en lengua inglesa, aparece information management, pero no la que podría ser su traducción literal, infonomics. Por ejemplo, según Cambridge Online:

"Information management: the process of collecting, organizing, storing, and providing information within a company or organization".

Si bien no aparece en ningún diccionario en español, sí pueden consultarse online varias definiciones que plantean algún matiz o variante sobre el mismo concepto:

- el Documentalista enredado (2007) define infonomía como: "gestión inteligente de la información";

- la web Estudiar en Cataluña (Generalitat de Catalunya, 2014) define infonomista como: "el profesional que tiene como misión la gestión inteligente de la información en cualquier tipo de organización";

- la web Barcelona treball (Ajuntament de Barcelona, s.f.): "profesional que tiene como misión la gestión inteligente de la información en cualquier tipo de organización. El infonomista es un perfil transversal que fusiona la cultura informática (cómo explotar las posibilidades de tecnologías de la información), la cultura informacional (cómo extraer valor de la información disponible) y la cultura de gestión (asegurar la transmisión y la capitalización del conocimiento adquirido en la organización)".

Infonomista no sustituiría a otras deno-

minaciones, sino que aportaría una visión de conjunto

Cabe señalar también que existe un término parecido en la forma, pero no equivalente en cuanto a significado, en inglés: infonomics. La Wikipedia en inglés (2015) atribuye su invención al analista de Gartner Doug Laney, citando un documento de 1999 en la actualidad out of print. Pero nótese que la misma Wikipedia le atribuye un significado más concreto y más vinculado a la economía, en comparación con infonomía, su aparentemente homólogo en español: "Infonomics is the theory, study and discipline of asserting economic significance to information".

De entrada, la propuesta original de infonomista aludía a una denominación genérica e independiente del puesto de trabajo y contexto del ejercicio profesional, a partir de la proyección de una competencia integral en la información como objeto central. No obstante la realidad muestra que 
este concepto no ha alcanzado plena difusión y reconocimiento a día de hoy. De hecho sigue sin existir un término inclusivo, en español o en inglés para designar en sentido amplio a los profesionales de la información, a las personas competentes para ello (Tejada-Artigas; Chacón-Jarén; Moreiro-González, 2014; Lawson; Kroll; Kowatch, 2010), en un contexto de escasa visibilidad académica y profesional (Moreiro-González, 2015; Taladriz-Mas, 2014; Abadal, 2013).

Hay un interés creciente por la información como objeto de estudio en un abanico cada vez más variado de disciplinas científicas

Respecto al espacio lingüístico y cultural anglosajón, esa misma cuestión permanece abierta como apunta Bates (2015), que toma nota de la confusión de nomenclatura entre distintas profesiones y disciplinas vinculadas a la información. Esta autora indica cómo las profesiones informacionales con más tradición, y sus respectivos nombres, están vinculadas a instituciones: biblioteca, archivo, museo. Y expone cómo posteriormente aparecen otros perfiles vinculados a la digitalización y a la provisión de servicios en organizaciones generalistas, tales como: gestor de conocimiento, records manager, etc. En este sentido, resulta interesante también constatar cómo la clasificación de profesiones de la Organización Internacional del Trabajo recoge claramente las profesiones ejercidas en instituciones, es decir, aquellas con más tradición, pero las profesiones más recientes no están estandardizadas al mismo nivel sino solamente citadas como ejemplos explicativos (ILO, 2012), contribuyendo todo ello a dificultar la visibilidad profesional.

A la vista de todo ello, vamos a plantear a continuación una discusión actualizada respecto al infonomista.

\section{Discusión}

Un aspecto, en principio favorable, de la denominación infonomista es su carácter independiente del contexto organizativo o del puesto de trabajo donde tiene lugar la gestión de la información en el actual panorama de digitalización, desma- terialización y deslocalización de la información (Marchionini; Moran, 2012). De entrada es una ventaja si pensamos en una organización genérica o en una disciplina integrada, como modo de superar la fragmentación que apunta Bates (2015). Pero también es cierto que por lo mismo puede producir recelo o suscitar escaso atractivo, ya sea entre quienes sienten el ejercicio de su profesión y disciplina fuertemente vinculados al contexto profesional de una institución y/o de un puesto de trabajo determinado, o bien entre quienes se identifican con alguna disciplina científica establecida que trate la información en alguna de sus facetas.

Otro aspecto interesante es el hecho de ser un término compacto, y susceptible de designar al experto, sea este un profesional en ejercicio o simplemente quien posee un conjunto de competencias relacionadas con la gestión de información, incluida una profunda comprensión de ésta como objeto central y de su estudio mediante el método científico.

\section{La débil conceptualización de "informa- ción" en el discurso coloquial y científi- co afecta negativamente a la visibilidad profesional}

Ahora bien, el tomar la información como objeto central tiene una serie de implicaciones diversas que conviene apuntar. Como primera observación el objeto central que propone el término es intangible. De entrada esto puede parecer una dificultad; sin embargo esa misma característica no ha sido óbice para que términos como psicólogo, por ejemplo, que se centra en un objeto percibido como intangible (el alma, la mente), estén plenamente introducidos.

Más preocupante es que la intangibilidad del objeto central, la información, crea confusión en el discurso cotidiano con otros conceptos relacionados como: comunicación, dato, documento, conocimiento (Ma, 2013; Boisot; Canals, 2004; Capurro; Hjorland, 2003). La consulta de diccionarios generales, ya sea en lengua española o en inglés, confirma esta confusión, como puede verse en las definiciones recogidas en la tabla 1.

Tabla 1. Definiciones de información en diccionarios generales

\begin{tabular}{|c|c|}
\hline Definiciones de información en inglés & Definición de información en español (Real Academia) \\
\hline $\begin{array}{l}\text { Information (Cambridge online): } \\
\text { - Facts about a situation, person, event, etc. } \\
\text { - News, facts or knowledge. }\end{array}$ & \multirow{3}{*}{$\begin{array}{l}\text { Información (Real Academia): } \\
\text { 1. f. Acción y efecto de informar. } \\
\text { 2. f. Oficina donde se informa sobre algo. } \\
\text { 3. f. Averiguación jurídica y legal de un hecho o delito. } \\
\text { 4. f. Pruebas que se hacen de la calidad y circunstancias necesarias en una persona } \\
\text { para un empleo u honor. U. m. en pl. } \\
\text { 5. f. Comunicación o adquisición de conocimientos que permiten ampliar o preci- } \\
\text { sar los que se poseen sobre una materia determinada. } \\
\text { 6. f. Conocimientos comunicados o adquiridos mediante una información. } \\
\text { 7. f. Biol. Propiedad intrínseca de ciertos biopolímeros, como los ácidos nucleicos, } \\
\text { originada por la secuencia de las unidades componentes. }\end{array}$} \\
\hline $\begin{array}{l}\text { Information (Oxford online): } \\
\text { - Facts provided or learned about something or someone. } \\
\text { - What is conveyed or represented by a particular arran- } \\
\text { gement or sequence. }\end{array}$ & \\
\hline $\begin{array}{l}\text { Information (Word reference-Random House): } \\
\text { - Knowledge gained through study, instruction, etc. } \\
\text { - Knowledge given or received about a particular fact; } \\
\text { news. } \\
\text { - Computer data at any stage of processing. }\end{array}$ & \\
\hline
\end{tabular}




\begin{tabular}{|c|c|}
\hline Retos & Oportunidades \\
\hline $\begin{array}{l}\text { - Puede provocar recelos si se entiende como sustituto de térmi- } \\
\text { nos que designan puestos de trabajo existentes vinculados a } \\
\text { contextos concretos (bibliotecario, community manager, records } \\
\text { manager, etc.). } \\
\text { - Escaso interés para ciertos colectivos que hoy gozan de un claro } \\
\text { reconocimiento como tales y pueden considerarlo redundante } \\
\text { (data scientists, informáticos). } \\
\text { - Confusión en el discurso cotidiano del objeto de su competen- } \\
\text { cia (información) con otros relacionados (dato, comunicación, } \\
\text { conocimiento, etc.). } \\
\text { - Minusvaloración en el discurso cotidiano del objeto de su com- } \\
\text { petencia (información) en comparación con otros conceptos } \\
\text { relacionados (dato, conocimiento). } \\
\text { - Débil conceptualización científica de la información. } \\
\text { Escasa cohesión del conglomerado de disciplinas y profesiones } \\
\text { vinculadas a la información. }\end{array}$ & $\begin{array}{l}\text { Define al experto, independientemente del contexto organizativo y } \\
\text { rol profesional concreto donde pueda eventualmente proyectar su } \\
\text { competencia. } \\
\text { - Término compacto. } \\
\text { - Concepto orientado a la gestión, a partir de una sólida base cientí- } \\
\text { fica. } \\
\text { - Creciente interés por la información como objeto de estudio y con- } \\
\text { cepto relevante, en un abanico cada vez más variado de disciplinas } \\
\text { científicas tanto tradicionales como nuevas. } \\
\text { - Potencial para cohesionar y dar sentido al conglomerado de dis- } \\
\text { ciplinas y profesiones actualmente existentes relacionadas con la } \\
\text { información. } \\
\text { - El movimiento académico de las Information Schools puede ayudar a } \\
\text { su aceptación. }\end{array}$ \\
\hline
\end{tabular}

Capurro y Hjorland (2003) resumen el concepto coloquial de información en lengua inglesa como "conocimiento comunicado". De hecho, las definiciones de la tabla 1 irían en esta línea, con más de una acepción corroborando este significado tanto en español como en inglés. En cuanto a la vinculación con disciplinas específicas, resulta remarcable en español la inclusión de acepciones directamente relacionadas con la disciplina jurídica y con la biología. En cambio en inglés, el significado de computer data en un cierto estadio de proceso, vincula con la informática.

La propia definición de información desde un punto de vista científico ha tenido diversos enfoques, resumidos por Gleick (2012), Floridi (2010), Capurro y Hjorland (2003). El significado de información ha tendido a hacerse más ambiguo con el paso del tiempo, tanto en el discurso científico como en el discurso cotidiano (Ma, 2012, 2013). Según este mismo autor, buena parte de las definiciones propuestas en el contexto de information science son demasiado ambiguas o pueden conllevar un uso metafórico, siendo por ello no operativas desde un punto de vista científico. Según su criterio, entre las definiciones a descartar estarían las que pretenden explicar la información como "dato", o como "dato procesado", por implicar la imposibilidad práctica de distinguir entre ciencia de la información e informática.

Que la conceptualización científica sea mejorable no impide que haya un creciente interés por parte de un amplio abanico de disciplinas científicas por la información como concepto y como objeto de estudio: biología, informática, economía, derecho, etc. En este sentido, la existencia desde 2004 de un movimiento académico como las Information Schools, que integra a decenas de facultades y departamentos académicos interesados en la información en sentido amplio, representa una oportunidad para integrar y potenciar el conocimiento científico sobre la información, y desarrollar en consecuencia el concepto de infonomista.

http://ischools.org

Otra faceta concierne al valor que se atribuye a la información. A menudo el discurso cotidiano considera la información como algo sin valor, gratuito, sobreabundante en comparación con otros conceptos generalmente vinculados a ella. Así, conocimiento se entiende en el lenguaje cotidiano como de mayor valor añadido, mientras que los datos y los documentos se ven como algo más concreto y tangible, y se entiende más fácilmente su potencial como fuente de valor. Información parece en peligro de quedarse en tierra de nadie entre unos y otros conceptos, sin una clara percepción del concepto en sí y de su potencial. En este sentido, es significativo el comentario que nos apuntaba en una conversación informal en 2011 Derek Stephens, de la Lougborough University: "Es difícil justificar la gestión del agua en un país con agua abundante; por razones análogas resulta difícil justificar la gestión de información en un mundo de información libre y gratuita". Esta frase expresa ideas en la línea de la problemática de justificación expuesta por Anglada (2014) para el caso específico de las bibliotecas.

\section{El movimiento académico de las Information Schools es una oportunidad para potenciar el concepto de infono- mista}

Un resumen de esta discusión, en coherencia con lo expuesto en este apartado, se muestra en la tabla 2, que apunta una selección de retos y oportunidades. Como se ve, no son pocos los retos para eventualmente consolidar y potenciar el concepto de infonomista, que a nuestro entender tendría sentido para dar respuesta a buena parte de las inquietudes e incertidumbres existentes tanto desde el punto de vista profesional como académico. Un punto clave sería avanzar en la definición del objeto central para el infonomista: la información como término especializado, tanto desde el punto de vista científico como profesional, sin olvidar atender al significado y uso del término en el discurso coloquial por parte del público general.

\section{Conclusiones}

Con su propuesta de infonomista, Cornella (1998) planteó definir un conjunto de competencias relacionadas con la gestión de información en sentido amplio (infonomía), a partir de la integración del conocimiento sobre la informa- 
ción de un conjunto de disciplinas científicas. Por tanto ello es independiente de la denominación de un puesto de trabajo concreto que se desempeñe en un determinado contexto. El infonomista como tal no sustituiría a otras denominaciones más concretas, sino que aportaría una visión de conjunto en relación a esas denominaciones.

A nuestro entender, a día de hoy las ideas fuerza de esa primera propuesta mantienen su potencial para responder a problemas tanto del mundo profesional como del académico. La integración del conocimiento científico actualmente disperso en una nueva disciplina (infonomía), podría poner la base para avances de dicho conocimiento, y también para un mayor impacto y visibilidad de los profesionales de la información. Desde un punto de vista académico, el movimiento de las Information Schools constituye una oportunidad para avanzar en esa línea y promocionar el concepto de infonomista.

La aceptación de infonomista podría cohesionar al conglomerado de disciplinas y profesiones vinculadas con la información

Ahora bien, para ello habría que resolver dos retos principales:

- la débil y dispersa conceptualización de información en el discurso científico y coloquial, que afecta también negativamente a la visibilidad profesional;

- los recelos de diversos colectivos, ya sea por su apego a denominaciones más específicas (archivero, bibliotecario, content curator, community manager, etc.) o porque podrían considerarlo redundante (data scientists, informáticos).

\section{Bibliografía}

Abadal, Ernest (2013). "La biblioteconomía y la documentación en la universidad española a principios del siglo XXI". Nuovi annali della scuola speciale per archiviste e bibliotecari, v. 27, pp. 211-228.

http://bd.ub.edu/pub/abadal/sites/bd.ub.edu.pub.abadal/ files/2013-abadal-NuoviAnnali.pdf

Ajuntament de Barcelona (s.f.). "Ficha ocupacional de infonomista". Barcelona treball.

http://w27.bcn.cat/porta22/es/fitxes/I/fitxa5989/infonomista. do

Anglada, Lluís (2014). "Are libraries sustainable in a world of free, networked, digital information?". El profesional de la información, v. 23, n. 6, pp. 603-611.

http://dx.doi.org/10.3145/epi.2014.nov.07

Bates, Marcia J. (2015). "The information professions: knowledge, memory, heritage". Information research, v. 20, n. 1, paper 655 .

http://InformationR.net/ir/20-1/paper655.html

Boisot, Max; Canals, Agustí (2004). "Data, information and knowledge. Have we got it right?". Journal of evolutionary economics, v. 14, n. 1, pp. 43-67.

http://www.uoc.edu/in3/dt/20388/

http://dx.doi.org/10.1007/s00191-003-0181-9
Capurro, Rafael; Hjorland, Birger (2003). "The concept of information". Annual review of information science and technology, v. 37, n. 8, pp. 343-411.

http://www.capurro.de/infoconcept.html http://dx.doi.org/10.1002/aris.1440370109

Cornella, Alfons (1998). “iEureka!: es la infonomía y somos infonomistas". El profesional de la información, v. 7, n. 10. http://www.elprofesionaldelainformacion.com/contenidos/1998/ octubre/aeureka_es_la_infonomia_y_somos_infonomistas.html

Cornella, Alfons (2000). Infonomia.com - La empresa es información. Bilbao: Deusto. ISBN: 9788423417605

Documentalista enredado (2007). "Qué es la infonomía". El documentalista enredado, 15 de septiembre.

http://www.documentalistaenredado.net/545/que-es-lainfonomia

Floridi, Marco (2010). Information: A very short introduction. Oxford: Oxford University Press. ISBN: 9780199551378 http://dx.doi.org/10.1093/actrade/9780199551378.001.0001

Generalitat de Catalunya (2014). "Infonomista". Estudiar en Cataluña.

http://queestudiar.gencat.cat/es/orientacio/itineraris/de_ que_tagradaria_treballar/humanistic_social/infonomista/

Gleick, James (2012). The information: a history, a theory, a flood. New York, NY: Vintage Books. ISBN: 9781400096237

International Labour Organization, ILO (2012). International standard classification of ocupations. Structure, group definitions and correspondence tables. ISBN: 9789221259534 http://www.ilo.org/wcmsp5/groups/public/---dgreports/--dcomm/---publ/documents/publication/wcms_172572.pdf

Lawson, Judy; Kroll, Joanna; Kowatch, Kelly (2010). The new information professional: your guide to careers in the digital age. New York: Neal-Schuman Publishers. ISBN: 978 1555706982

Ma, Lai (2012). "Meanings of information: The assumptions and research consequences of three foundational LIS theories". Journal of the American Society for Information Science and Technology, v. 63, n. 4, pp. 716-723. http://dx.doi.org/10.1002/asi.21711

Ma, Lai (2013). "Is information still relevant". Information research, v. 18, n. 3, paper C33.

http://InformationR.net/ir/18-3/colis/paperC33.html

Marchionini, Gary; Moran, Barbara (eds.) (2012). Information professionals 2050: Educational possibilities and pathways. School of Information and Library Science. University of North Carolina at Chapell Hill.

http://sils.unc.edu/sites/default/files/publications/InformationProfessionals-2050.pdf

Moreiro-González, José-Antonio (2014). “Aún en torno a la formación universitaria, el empleo y la recesión". Anuario ThinkEPI, v. 8, pp. 15-23.

Moreiro-González, José-Antonio (2015). "Al hilo de las noticias sobre planes de estudio y mercado de trabajo". Anuario ThinkEPI, v. 9, pp. 13-24.

http://dx.doi.org/10.3145/thinkepi.2015.02 
Moreiro-González, José-Antonio; Azcárate-Aguilar-Amat, Pilar; Marzal-García-Quismondo, Miguel-Ángel; TejadaArtigas, Carlos-Miguel; Vergueiro, Waldomiro C. S. (2008). "Desarrollo profesional y opinión sobre la formación recibida de los titulados universitarios en información y documentación de las universidades públicas de Madrid (20002005)". El profesional de la información, 2008, v. 17, n. 3, pp. 261-272.

http://recyt.fecyt.es/index.php/EPI/article/view/epi.2008. may.02

http://dx.doi.org/10.3145/epi.2008.may.02

Ortiz-Repiso, Virginia; Calzada-Prado, Javier; Aportela-Rodríguez, Ivett M. (2013). “¿Qué está pasando con los estudios universitarios de biblioteconomía y documentación en España?". El profesional de la información, v. 22, n. 6, pp. 505-514. http://dx.doi.org/10.3145/epi.2013.nov.02

Taladriz-Mas, Margarita (2014). “Gestores de información y reconocimiento social". BiD: textos universitaris de biblioteconomia i documentació, n. 32.

http://dx.doi.org/10.1344/BiD2014.32.2

Tejada-Artigas, Carlos-Miguel; Chacón-Jarén, Sandra; Moreiro-González, José-Antonio (2014). “Mercado de trabajo en información y documentación y crisis económica en España: una aproximación a partir de las ofertas publicadas en IweTel entre 2008 y 2013". BiD: textos universitaris de biblioteconomia i documentació, n. 32.

http://dx.doi.org/10.1344/BiD2014.32.15

Wikipedia (2015). Infonomics.

https://en.wikipedia.org/wiki/Infonomics

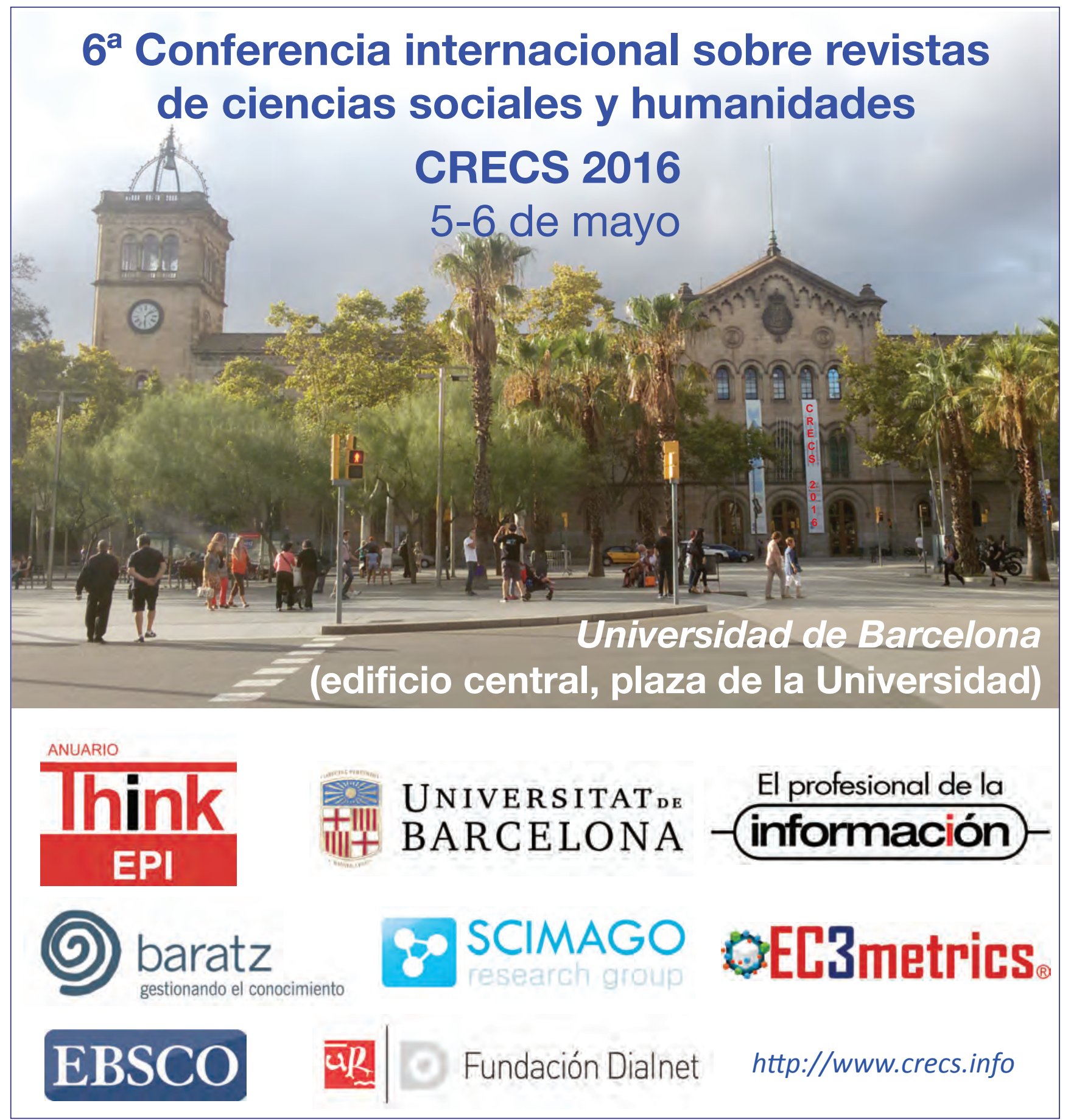

